\title{
S Research Square

\section{Simultaneous Daily Platelet Count And Presepsin Measurement From The Time Of ICU Admission May Be Useful For Predicting In-Hospital Mortality Of Patients With Severe COVID-19}

Hiroyasu Ishikura ( $\square$ ishikurah@fukuoka-u.ac.jp )

Fukuoka University

Junichi Maruyama

Fukuoka University

Maiko Nakashio

Fukuoka University

Kota Hoshino

Fukuoka University

Shinichi Morimoto

Fukuoka University

Yoshito Izutani

Fukuoka University

Junta Noake

Fukuoka University

Takahiro Yamagaito

Sysmex Product Marketing

Maho Yoshida

Sysmex Scientific Affairs

Taisuke Kitamura

Fukuoka University

Yoshihiko Nakamura

Fukuoka University

\section{Research Article}

Keywords: SARS-CoV-2, COVID-19, coagulopathy, platelet count, presepsin, prognosis

Posted Date: February 25th, 2022

DOI: https://doi.org/10.21203/rs.3.rs-1383326/v1 
License: (c) (i) This work is licensed under a Creative Commons Attribution 4.0 International License. Read Full License 


\section{Abstract}

Background: coronavirus disease 2019 (COVID-19) patients are frequently complicated with COVID-19 associated coagulopathy. This time, we investigated whether there was a relationship between the CAC pattern and the risk of in-hospital death in severe COVID-19 patients.

Methods: We enrolled 54 severe COVID-19 adult patients admitted to intensive care unit (ICU). Patients were divided into two groups whether in-hospital death (non-survivor) or not (survivor). We measured not only various coagulation/fibrinolysis markers, but inflammation/immunoresponse markers which were cytokines or chemokines, and alveolar/myocardial damage markers in peripheral blood every day for 8 ICU days. Univariate logistic regression analysis and receiver operating characteristic curve (ROC) analysis of the area under the curve (AUC) were used to identify the optimal combination of biomarkers to predict in-hospital death.

Results: Among all 54 patients, the patients were classified into the survivor $(n=40)$ and non-survivor $(n=$ 14) groups. We found that the platelet count decreased significantly and presepsin (P-SEP) increased significantly in non-survivor group compared to survivor group from the time of ICU admission. Using univariate logistic regression analysis, platelet count and P-SEP are found to be predictive of in-hospital mortality and ROC analysis using these two markers could further confirm this result ( $A U C=0.832$; sensitivity: $64.3 \%$, specificity: $90.0 \%$ ). The cut-off values for in-hospital mortality for these markers were $153 \times 10^{3} / \mu \mathrm{L}$ in platelet count and $714 \mathrm{pg} / \mathrm{mL}$ in P-SEP. The patients were consequently classified into the following 3 groups: (1) High risk $(n=7)$, platelet count $\leq 153 \times 10^{3} / \mu \mathrm{L}$ and P-SEP $\geq 714 \mathrm{pg} / \mathrm{mL}$; (2) Low risk $(n=27)$, platelet count $>190 \times 10^{3} / \mu \mathrm{L}$ and P-SEP $<714 \mathrm{pg} / \mathrm{mL}$; (3) Medium risk $(\mathrm{n}=20)$, areas other than (1) and (2). And the 90-day mortality increased significantly as the risk of the group increases $(p<0.01)$. Additionally, the platelet count was lower and P-SEP level was higher in the non-survivor group than in the survivor group on ICU days throughout the 8 days.

Conclusions: Simultaneous daily platelet counts and P-SEP measurements from the time of ICU admission would be a useful method for predicting in-hospital mortality.

\section{Background}

In December 2019, a novel beta coronavirus named severe acute respiratory syndrome coronavirus (SARS-CoV)-2, which was thought to originate in Wuhan, China, emerged as a new human pathogen for viral pneumonia [1]. SARS-CoV-2 has led to a global outbreak of coronavirus disease 2019 (COVID-19) and was declared a pandemic by the World Health Organization on 11 March 2020 [2]. Substantial progress in clinical research has led to a better understanding of SARS-CoV-2 and the management of COVID-19. However, limiting the continuing spread of this virus and its variants has become an issue of increasing concern. SARS-CoV-2 continues to wreak havoc worldwide, with many countries enduring a third or fourth wave of outbreaks of this viral illness, attributed mainly to the emergence of mutant variants of the virus [3]. Several variants of SARS-CoV-2, including Alpha (B.1.1.7), Beta (B.1.351), and 
Gamma (P.1), have been reported during this pandemic. The B.1.617.2 (Delta) variant, which was first detected in India in December 2020, has become the most prevalent and commonly reported variant in the world since mid-April 2021. As of 19 May 2021, this variant had been detected in 43 countries across 6 continents [4].

The viral spike (S) protein of SARS-CoV-2 binds to angiotensin-converting enzyme (ACE) 2 as a cellular receptor. This binding provides viral entry into host cells and plays a crucial role in the pathogenesis of COVID-19. The ACE2 receptor is highly expressed in type II lung cells [5]. Therefore, the lungs are the primary target organ of SARS-CoV-2, and acute lung injury and acute respiratory distress syndrome are the most common complications observed in patients with COVID-19 [6]. Although the respiratory system is the principal target for SARS-CoV-2 as described above, it can affect other major organ systems, such as the gastrointestinal tract, and hepatobiliary, cardiovascular, renal, and central nervous systems. SARSCoV-2-induced organ dysfunction may be explained by either one or a combination of the proposed mechanisms, such as direct viral toxicity, ischemic injury caused by vasculitis, thrombosis or thromboinflammation, immune dysregulation, and renin-angiotensin-aldosterone system dysregulation [7].

The measurement of sepsis biomarkers represents a cornerstone in the risk assessment of patients with severe infectious diseases and/or sepsis because they can help predict clinical progression and guide therapeutic management [8]. Not surprisingly, patients with severe COVID-19 are frequently complicated by coagulation/fibrinolysis abnormalities [9-11]. These abnormalities are called COVID-19-associated coagulopathy (CAC) because the disease arises from infection with SARS-CoV-2. An elevated D-dimer concentration, which is the most common coagulation abnormality in COVID-19 (found in $45 \%$ of patients), is an independent risk factor for death [12]. Additionally, thrombocytopenia occurs more often in patients with severe disease and may be correlated with increased mortality [13]. Most patients with COVID-19 have a normal or mildly deviated prothrombin time (PT) and activated partial thromboplastin time (APTT) on presentation, but these factors are not reliably associated with the disease severity [12].

This study aimed to determine the characteristics of CAC using biomarkers of coagulation/fibrinolysis, inflammation/immunoresponse, and alveolar/myocardial damage, and to identify prognostic markers in patients with serious COVID-19 from these biomarkers. To date, there have been few reports on the time course of coagulation/fibrinolysis or inflammatory abnormalities and the risk of death, with a focus on patients with severe COVID-19. Therefore, we measured these biomarkers daily to determine how they changed over time.

\section{Methods}

This retrospective cohort study was conducted at the Extracorporeal Membranous Oxygenation (ECMO) Center, Fukuoka University Hospital, Fukuoka, Japan, which is a 915-bed referral and tertiary hospital, from April 2020 to May 2021. We obtained informed consent from the proxy in accordance with the Declaration of Helsinki for publication of this study. Patients with severe COVID-19 aged $\geq 20$ years who were diagnosed with SARS-CoV-2 infection detected by reverse transcription-polymerase chain reaction 
from a nasopharyngeal swab sample were enrolled in the study. Severe COVID-19 was defined as the condition in which patients were admitted to the intensive care unit (ICU) for COVID-19-induced sepsis with acute respiratory distress syndrome and they required mechanical ventilation management or additional ECMO management. Patients were evaluated for the presence of sepsis according to the Sepsis-3 diagnosis criteria [14] and for the presence of acute respiratory distress syndrome according to the Berlin definition [15]. The disseminated intravascular coagulation (DIC) scoring system of the Japanese Association for Acute Medicine (JAAM) was used for the diagnosis of DIC. The JAAM DIC diagnostic algorithm for scoring DIC includes the following variables: platelet count, PT, fibrin/fibrinogen degradation product (FDP) level, and systemic inflammation response syndrome criteria (Additional files; Supplemental Tables 1 and 2). The details of the algorithm have been published elsewhere [16]. DIC was defined as a score of $\geq 4$. We evaluated three major DIC scoring systems, which comprised the JAAM DIC criteria, the International Society on Thrombosis and Haemostasis (ISTH) overt DIC criteria (Additional file; Supplemental Table 1) [17], and the Japanese Ministry of Health and Welfare (JMHW) DIC criteria (Additional file; Supplemental Table 1) [18]. The illness severity was evaluated by the Acute Physiology and Chronic Health Evaluation (APACHE) II score [19]. The APACHE II score assesses the illness severity in critical patients admitted to an ICU on the basis of routine physiologic measurements, age, and previous health status. This score is used to predict the outcomes of critical illnesses. Organ failure was assessed by the Sequential Organ Failure Assessment (SOFA) score [20]. The SOFA score estimates organ dysfunction related to various disease statuses, especially sepsis, and is calculated using readily available measurements to quantify dysfunction of the six major organs. The SOFA score is also useful for evaluating the morbidity and mortality of critical illnesses.

Patients with severe COVID-19 were divided into the survivor group and the non-survivor group. The survivor group was defined as the absence of in-hospital death and the non-survivor group as the presence of in-hospital death.

\section{Study Procedures}

In patients with severe COVID-19, blood samples were routinely collected for measuring markers, and there was no lack of data on ICU admission and subsequent 8 days in this study. We collected peripheral blood and measured coagulation/fibrinolysis biomarkers, such as the platelet count, the PT-international normalized ratio (PT-INR), the APTT, FDP, D-dimer, thrombin-antithrombin complex (TAT), plasmin a2plasmin inhibitor complex, antithrombin (AT), protein C, thrombomodulin (TM), soluble fibrin (SF), and plasminogen activator inhibitor (PAI)-1. We also measured inflammation/immunoresponse markers, which comprised the white blood cell (WBC) count, C-reactive protein (CRP), presepsin (P-SEP), procalcitonin (PCT), interleukin (IL)-6, IL-10, IL-18, monokine induced by interferon- $\gamma$, and macrophage inflammatory protein. We also measured Krebs von den Lungen (KL)-6 and surfactant protein A (SP-A) concentrations as markers of alveolar damage [21, 22], and N-terminal pro B-type natriuretic peptide (NTproBNP) and troponin $\mathrm{T}(\mathrm{TnT})$ concentrations as markers of myocardial damage [23, 24]. Additionally, we measured total bilirubin, creatinine, lactate dehydrogenase, and ferritin concentrations. 
Platelet and WBC counts were measured in whole blood using the XT-1800i analyzer (Sysmex Co., Kobe, Japan). The PT, the activated partial thromboplastin time, fibrin/fibrinogen degradation products, D-dimer concentrations, AT activity, protein C activity, and SF concentrations were measured in plasma using the CP 3000 analyzer (Sekisui Medical, Tokyo, Japan). TM and PAI-1 concentrations were measured using the STACIA system (LSI Medience Co., Tokyo, Japan). CRP concentrations were measured by the CRPLatex (II) X2 Seiken Assay Kit (Denka Seiken Co. Ltd., Tokyo, Japan). P-SEP concentrations were measured using the HISCL-5000 fully automated immunochemistry analyzer, which uses chemiluminescence enzyme immunoassay methodology (Sysmex Co., Kobe, Japan). PCT concentrations were measured by the Elecsys BRAHMS PCT assay (Roche Diagnostics, Tokyo, Japan). IL-6, IL-10, IL-18, monokine induced by interferon- $\gamma$, and macrophage inflammatory protein-1 a concentrations were also measured using the HISCL-5000 analyzer (Sysmex Co., Kobe, Japan). Additionally, KL-6, SP-A, NT-proBNP, and TnT concentrations were measured using HISCL-5000 analyzer (Sysmex Co., Kobe, Japan). Total bilirubin, creatinine, and lactate dehydrogenase concentrations were measured using the TBA-c16000 (Canon Medical Systems Co., Tochigi, Japan), and ferritin concentrations were measured using the Cobas 8000 (Roche Diagnostics). The PT-INR was calculated using the following formula: INR = (patient's $\mathrm{PT} /$ normal $\mathrm{PT})^{\mid \mathrm{SI}}$, where normal PT represents the average of the mean normal PT range in laboratory results and ISI is the International Sensitivity Index, which is a correction coefficient for thromboplastin in commercial kits calculated according to international reference samples.

\section{Statistical analysis}

Unless otherwise indicated, all data are expressed as the median (interquartile range). SPSS $15.0 \mathrm{~J}$ (SPSS Inc., Chicago, IL, USA) was used for statistical analyses. Nonparametric statistical tests were used because these tests are more appropriate than traditional parametric tests for analysis of data sets with a high variability. Differences in biomarker levels between the two patient groups (survivors and nonsurvivors) were analyzed using the Mann-Whitney U test. Comparisons between three or more groups were carried out using the Bonferroni Method.

The standard error of the receiver operating characteristic curve (ROC) analysis was calculated using the formula described by Hanley and McNeil [25]. A univariate logistic regression model analysis and ROC analysis were used to assess the relationships between in-hospital death and various biomarkers. The level of statistical significance was set at $P<0.05$.

\section{Results}

\section{Population characteristics}

Fifty-four consecutive patients with severe COVID-19 were enrolled from April 2020 to August 2021, and all patients were included in the analysis. The median age of the patients (44 men; 10 women) was 60 (54-68) years. Among all patients, 40 were classified into the survivor group and 14 were classified into the non-survivor group (survival rate: 74.1\%) (Table 1). The non-survivor group was significantly older 
than the survivor group $(P<0.01)$. Moreover, the non-survivor group had significantly higher APACHE II $(P$ $<0.01)$ and SOFA $(P=0.02)$ scores than the survivor group (Table 1$)$. The non-survivor group also had significantly higher rates of continuous renal replacement therapy $(P<0.01)$ than the survivor group (Table 1). The non-survivor group had a significantly lower body mass index $(P=0.03)$, total percentage of the lymphocyte/WBC ratio $(P<0.01)$, and hemoglobin level $(P=0.04)$ than the survivor group (Table 1$)$. The scores for the JAAM DIC, the ISTH overt DIC, and the JMHW DIC scoring systems were not significantly different between the non-survivor group and the survivor group (Table 1). We also calculated the DIC positivity rate using major DIC scoring systems, and found that the DIC positivity rate in all DIC scoring systems was not significantly different between the two groups (Table 1).

\section{Distribution Of Various Molecular Biomarkers}

Differences in baseline biomarker levels, such as coagulation/fibrinolysis, inflammation/immunoresponse, and alveolar/myocardial damage markers, at ICU admission between the survivor and non-survivor group are shown in Table 2. With regard to coagulation/fibrinolysis markers, the non-survivor group had a significantly lower platelet count $(P<0.01)$ than the survivor group at ICU admission. With regard to inflammation/immunoresponse markers, the non-survivor group had significantly higher P-SEP $(P=0.04)$ and IL-10 $(P<0.01)$ concentrations than the survivor group. With regard to alveolar/myocardial damage markers, the non-survivor group had significantly higher KL-6 ( $P=$ $0.03)$ concentrations than the survivor group.

\section{Association Of Biomarker Levels With In-hospital Death}

We then performed univariate logistic regression model analysis to evaluate the predictive power for inhospital mortality using the above-mentioned biomarkers that were significant $(P<0.05)$ between the survivor and non-survivor groups on ICU admission. The platelet count, P-SEP, and KL- 6 showed predictive power, with $P<0.05$ as the cut-off for significance (Table 3). We then performed an ROC analysis using the platelet count, P-SEP, and KL-6, and calculated the area under the curve (AUC) reported as a quantification of this analysis. The AUC of the platelet count was 0.786 , that of P-SEP was 0.687 , and that of KL- 6 was 0.700 (Table 3). Additionally, we performed an ROC analysis including the AUC using these three markers to identify the optimal combination for assessing in-hospital mortality. This analysis showed that a combination of the platelet count and P-SEP concentrations at the time of ICU admission may be an optimal combination to predict patients' outcomes. When the platelet count and P-SEP concentrations were selected as predictive markers for in-hospital death, in-hospital death was able to be predicted with a high probability, with an AUC $=0.832$ (sensitivity: $64.3 \%$, specificity: $90.0 \%$ ). The AUCs of other combinations of the platelet count and KL-6, and P-SEP and KL-6, were 0.813 and 0.775 , respectively.

The cut-off values of in-hospital mortality for these markers were $153 \times 10^{3} / \mu \mathrm{L}$ for the platelet count (sensitivity: $64.3 \%$, specificity: $82.5 \%$ ) and $714 \mathrm{pg} / \mathrm{mL}$ for P-SEP concentrations (sensitivity: $64.3 \%$, 
specificity: $77.5 \%)$.

Selection of a multi-marker panel and development of a COVID-19 severity classification algorithm

In this study, the following biomarkers were the optimal biomarker panel for predicting in-hospital mortality: (1) platelet count (a coagulation marker) and (2) P-SEP (an inflammatory biomarker). The patients enrolled in this study were consequently divided into four panels according to these cut-off values (Fig. 1). The in-hospital mortality rate of each panel was as follows: 1) platelet count $\leq 153 \times 10^{3} /$ $\mu \mathrm{L}$ and P-SEP concentrations $\geq 714 \mathrm{pg} / \mathrm{mL}, 85.7 \%(7 / 6) ; 2$ ) platelet count $>153 \times 10^{3} / \mu \mathrm{L}$ and P-SEP concentrations $\geq 714 \mathrm{pg} / \mathrm{mL}, 27.3 \%(3 / 11) ; 3)$ platelet count $\leq 190 \times 10^{3} / \mu \mathrm{L}$ and P-SEP concentrations < $714 \mathrm{pg} / \mathrm{mL}, 33.3 \%$ (3/9); and 4) platelet count $>190 \times 10^{3} / \mu \mathrm{L}$ and P-SEP concentrations $<714 \mathrm{pg} / \mathrm{mL}$, $7.4 \%(2 / 27)$. We then classified the patients into the following three categories according to the platelet count and P-SEP concentrations as follows: ( 1$)$ high risk $(n=7)$, platelet count $\leq 153 \times 10^{3} / \mu \mathrm{L}$ and P-SEP concentrations $\geq 714 \mathrm{pg} / \mathrm{mL}$; (2) medium risk $(\mathrm{n}=20)$, platelet count $>153 \times 10^{3} / \mu \mathrm{L}$ and P-SEP concentrations $\geq 714 \mathrm{pg} / \mathrm{mL}$, or platelet count $\leq 153 \times 10^{3} / \mu \mathrm{L}$ and P-SEP concentrations $<714 \mathrm{pg} / \mathrm{mL}$; and (3) low risk $(n=27)$, platelet count $>153 \times 10^{3} / \mu \mathrm{L}$ and P-SEP concentrations $<714 \mathrm{pg} / \mathrm{mL}$ (Fig. 1$)$.

The in-hospital mortality rate of patients in each group was $85.7 \%(6 / 7)$ in the high-risk group, $30.0 \%$ $(6 / 20)$ in the medium-risk group, and $7.4 \%(2 / 27)$ in the low-risk group. The in-hospital mortality significantly increased as the risk of the group increased (Fig. 1).

\section{Illness Severity According To The Classification Of In- hospital Death}

We propose the COVID-19 severity panel as a clinically useful numerical representation of the results from our multi-marker panel, which includes the platelet count and P-SEP panel testing. Additionally, to evaluate the diagnostic accuracy of this panel, we investigated whether this panel was able to stratify patients according to physiological severity, the degree of organ failure, age, and 90-day mortality. When patients were categorized into three groups according to the severity defined in this panel, the APACHE II score and the SOFA score on ICU admission significantly increased as the COVID-19 severity increased (Fig. 2). Age was not significantly different between the three groups. However, the risk of the group increased as age increased. The Kaplan-Meier curve for 90-day survival by each of the three groups derived from the panel is shown in Fig. 3. The survival rate was significantly higher in the low-risk group than in the medium-risk group and high-risk group (both $P<0.01$ by the log-rank test).

\section{Time Course Of The Distribution Of Various Biomarkers}

The daily changes in the distribution of various molecular biomarkers up to the 8th day in the ICU are shown in Fig. 4 (11 coagulation/fibrinolytic markers) and Fig. 5 (8 inflammation/immune response 
markers, 2 alveolar damage markers, and 2 myocardial damage markers).

\section{Coagulation/fibrinolysis markers}

Only the platelet count was significantly lower in the non-survivor group than in the survivor group on all 8 days. TAT concentrations were significantly higher in the non-survivor group than in the survivor group only on Day 2. TAT, plasminogen activator inhibitor-1, and soluble fibrin concentrations were significantly different between the survivor and non-survivor groups only on 1 or 2 days throughout the 8 days. However, this result was not helpful in distinguishing both groups early in the ICU stay. There were no significant differences in the other markers between the two groups over the 8-day period, and the initial values of these coagulation/fibrinolysis markers in the ICU stay were not predictive of the patients' outcomes (Fig. 4).

\section{Inflammation/immunoresponse markers}

Only IL-10 concentrations were significantly higher in the non-survivor group than in the survivor group on all 8 days. P-SEP concentrations were significantly higher in the non-survivor group than in the survivor group on 4 of the 8 days, excluding days $2,3,4$, and 6 . PCT concentrations were significantly higher in the non-survivor group than in the survivor group on 4 of the 8 days, excluding days 1 to 4 . IL-6 and IL-18 concentrations were significantly different between the survivor and non-survivor groups on only 1 or 2 days throughout the 8 days. Other markers (CRP, monokine induced by interferon- $\gamma$, and macrophage inflammatory protein-1a) were not significantly different between the two groups over the 8-day period, and the initial values of these markers in the ICU stay were not predictive of the patients' outcomes (Fig. 5).

\section{Alveolar/myocardial damage markers}

KL- 6 concentrations were significantly higher in the non-survivor group than in the survivor group on 3 of the 8 days. There was no significant difference in SP-A concentrations between the two groups over the 8day period. NT-proBNP concentrations were significantly higher in the non-survivor group than in the survivor group on 6 of the 8 days, excluding days 1 and 2. TnT concentrations were not significantly different between the two groups over the 8-day period (Fig. 5).

\section{Discussion}

The new infectious disease COVID-19 currently has no established curative treatment, and has a high mortality rate when it becomes severe. Therefore, the identification of biomarkers that can predict not only the severity but also the prognosis of this disease, is important for guiding the treatment direction and saving patients' lives.

Recent studies on COVID-19 have shown that levels of certain coagulation/fibrinolysis markers, inflammatory markers, or cytokines are good indicators for predicting a poor prognosis or severity of this disease [26-30]. This study showed that a decrease in the platelet count, which is a coagulation marker, 
and an increase in concentrations of P-SEP, which is an inflammation marker, were good indicators for predicting a poor prognosis and the time course of a critical condition. We also found that the prognosis of patients with severe COVID-19 was significantly worse if inflammation and coagulation were enhanced simultaneously. Additionally, measuring these two markers simultaneously and applying those levels to our proposed panel provided a high probability of diagnosing in-hospital death. To date, there have been no reports limited to critically ill patients with COVID-19. Our findings may be useful for ICU management of patients with COVID-19, especially those in a critical condition.

Thrombocytopenia is frequently the initial feature in sepsis, and it may be followed by the prolongation of global coagulation [31]. In patients with sepsis, thrombocytopenia is associated with an increase in the rate of mortality [32,33]. Additionally, mortality of these patients is associated with prolonged thrombocytopenia and the absence of a relative increase in the platelet count [32].

In a previous study, thrombocytopenia was detected in 5-41.7\% of patients with COVID-19 [34]. Thrombocytopenia also occurs more often in patients with COVID-19 with severe disease and is potentially correlated with an increased mortality rate $[13,26]$. Measurement of the platelet count is a simple, economic, rapid, and commonly available laboratory parameter [13]. Therefore, we believe that daily measurement of the platelet count can discriminate between patients with COVID-19 with and without severe disease.

In this study, patients with severe COVID-19 who had a low platelet count on ICU admission (cut-off value: $153 \times 10^{3} / \mu \mathrm{L}$ ) that remained low without increasing throughout the first week of stay in the ICU had a poor prognosis. This finding suggests that daily measurement of the platelet count is a useful and essential tool for predicting in-hospital mortality. Additionally, this cut-off value is similar to the predicted value for bleeding complications reported by Al-Samkari et al. [35].

P-SEP is known as soluble CD14 subtype. P-SEP is a 13-kDa protein and is a truncated $\mathrm{N}$-terminal fragment of CD14, which is the receptor for lipopolysaccharide/lipopolysaccharide binding protein complexes [36,37]. This biomarker is mainly synthesized and released by cells of the monocyte/macrophage lineage in response to a vast array of infections [38]. P-SEP concentrations increase in the blood of septic patients. The measurement of P-SEP is reported to be useful for the initial diagnosis of sepsis, evaluating the severity of sepsis, risk stratification, and monitoring clinical responses to therapeutic interventions [39-42].

Recently, studies have reported that elevated concentrations of P-SEP may be a biomarker in the prognostic assessment of patients with COVID-19 [27, 28]. Schirinzi et al. [29] reported that P-SEP and IL6 concentrations reflect the clinical course of COVID-19. Additionally, in a population of patients with COVID-19 and acute respiratory failure in the Emergency Department, P-SEP and troponin I are accurate predictors of 30-day mortality [43]. Additionally, P-SEP is highly specific and may enable the early identification of patients who could benefit from more intensive care as soon as they enter the Emergency Department [43]. A pooled analysis also showed a positive difference in P-SEP values between patients 
with or without severe/critical COVID-19 illness. A previous study showed that P-SEP values were increased by 2.74-fold in patients with COVID-19 with severe/critical illness compared with those without COVID-19 [44]. To date, there have been no reports of elevated P-SEP concentrations during viral infection. There have been a few reports that showed no increase in P-SEP concentrations under an influenza virus infection [45]. However, the cases were not severe. In our study of patients with severe COVID-19, survivors had significantly higher P-SEP concentrations than non-survivors throughout the first week of their stay in the ICU. We speculate that the reason for this finding is as follows. In patients with COVID-19, macrophages actively phagocytose SARS-CoV-2 to eliminate this virus that has invaded the body. Additionally, the active and robust invasion of SARS-CoV-2 into macrophages may contribute to an increase in P-SEP concentrations in the bloodstream. The ACE2 receptor is expressed on the surface of macrophages, and the SARS-CoV-2 spike protein binds to this receptor, eventually allowing the virus to invade macrophages. Tavazzi et al. showed that viral particles were observed within macrophages from a patient with COVID-19 and severe pulmonary inflammation and cardiogenic shock requiring ECMO [46]. Therefore, we suspect that SARS-CoV-2 actively invades macrophages, causing immunodeficiency and worsening the prognosis of patients. At the same time, blood P-SEP concentrations may be elevated.

In this study, we found that patients with severe COVID-19 who had high P-SEP values on ICU admission (cut-off value: $714 \mathrm{pg} / \mathrm{mL}$ ) that remained high without decreasing throughout the first week of their stay in the ICU had a poor prognosis. Previous reports showed a predicted cut-off value of $600-800 \mathrm{pg} / \mathrm{mL}$ for the diagnosis of sepsis, which was in close agreement with the cut-off value for in-hospital death in patients with severe COVID-19 [39-42].

In our study, PCT did not reflect the clinical course of COVID-19. Therefore, PCT cannot be used as an indicator for predicting a poor prognosis and the time course of a critical condition. The reasons why PCT concentrations were not greatly elevated in both groups and why there was no difference in PCT concentrations between both groups might be as follows. PCT is usually produced in parenchymal cells, such as the lung, liver, kidney, and muscle, owing to an increase in inflammatory cytokines (e.g., IL-1 and/or tumor necrosis factor (TNF)- $a$ ) when patients develop sepsis or a serious inflammatory reaction due to a bacterial infection $[47,48]$. However, the majority of cytokines that are associated with the cytokine storm in viral infections, such as IL- 6 and interferon- $\gamma$, are only significantly elevated in the late stage of severe COVID-19 illness [49]. Additionally, PCT is difficult to increase in viral and fungal infections [50]. Therefore, SARS-CoV-2 infections, in contrast to bacterial infection, usually induce only a modest and delayed increase in circulating PCT concentrations [47]. These findings suggest that, in patients with COVID-19 and high PCT concentrations, high PCT concentrations may be associated with bacterial co-infection. Because of the association of PCT concentrations with bacterial co-infection and severe disease, serially testing of calcitonin levels has been recommended, particularly in ICU patients [51, 52]. Serial PCT measurement may play a role in predicting evolution toward a more severe form of disease $[53,54]$.

Patients with COVID-19 show markedly increased inflammatory and anti-inflammatory cytokines, such as IL-6, IL-2R, IL-10, and TNF-a concentrations [30]. Among them, IL-10 concentrations reflect the severity of 
patients, and IL-10 concentrations are significantly higher in the severe group of patients than in the moderate group $[49,55,56]$. In our study, IL-10 concentrations were significantly higher in the non-survivor group than in the survivor group on all 8 days. A cytokine storm in patients with COVID-19 is similar to that previously observed in patients with severe acute respiratory syndrome infected by SARS-CoV. However, a unique feature of the COVID-19 cytokine storm is the dramatic elevation of IL-10 concentrations in severe/critically ill patients $[55,57-60]$. IL-10 concentrations are elevated earlier than IL-6 concentrations in patients with COVID-19 [61].

How SARS-CoV-2 infection differs from SARS-CoV in its capacity to stimulate IL-10 expression is currently unknown. However, while IL-10 is usually considered an anti-inflammatory cytokine that plays a role in suppressing an excess immune system, IL-10 may play a pro-inflammatory and immune-activating role in the pathogenesis of COVID-19 $[55,62]$.

In this study, concentrations of NT-proBNP, which is a marker of myocardial damage, were significantly higher in the non-survivor group than in the survivor group on 6 of the 8 days, excluding Day 1. However, KL- 6 and SP-A, which are markers of alveolar injury, were not suitable for predicting the outcome. We initially expected that KL-6 and SP-A would be better predictors of the outcome than NT-proBNP, but this was not found. A recent review article described that cardiac injury caused by COVID-19 infection might be an important cause of severe clinical phenotypes [54]. This review also suggested that myocardial damage is closely related to the severity of the disease and even the prognosis in patients with COVID-19. A review article by Ali et al. [63] described the following. ACE2 was first cloned from the human heart, but not bronchi, and was found to be highly expressed throughout the endothelia of coronary and renal blood vessels. This finding suggests important biological functions of ACE2 in the cardiovascular system. On the basis of these findings, we suspected that NT-proBNP may be a better predictor of the patients' outcomes than KL-6 and SPA. However, the levels of biomarkers of myocardial injury, such as NT-proBNP and $\mathrm{TnT}$, are affected by many factors, such as infection, hypoxia, and renal function. Therefore, the potential for false positives for myocardial injury in patients with COVID-19 must be considered [54]. Consequently, the patients' outcomes should not be predicted with just one measurement of NT-proBNP.

Generally, concentrations of D-dimer, which is a breakdown product of cross-linked fibrin, are correlated with the disease severity and predict the risk of thrombosis, the requirement for ventilator support, and mortality [64]. However, in our study, D-dimer was not a useful predictive marker of in-hospital mortality. We suspect that the reason for this finding is that when patients with COVID-19 develop a severe condition and need to be admitted to the ICU, D-dimer concentrations remain high, regardless of whether they die in the hospital.

This study has limitations. First, this was a retrospective study and the measurement of each biomarker for each patient was at different times from the beginning of COVID-19 infection. Second, approximately $55 \%$ of patients were administered anticoagulant, mainly unfractionated heparin, after ICU admission for performing ECMO. Third, because this study focused on measurable markers in our hospital, few data on 
other markers were examined. Fourth, this study was a relatively short-term study in Fukuoka, Japan and was a single-center study. Therefore, further research is required to confirm our findings.

\section{Conclusions}

This study suggests that measurement of the platelet count and P-SEP concentrations in blood individually and in combination may be useful for predicting the prognosis of patients with severe COVID19 and for guiding treatment strategies.

\section{Abbreviations}

ACE: angiotensin-converting enzyme; APACHE: Acute Physiology and Chronic Health Evaluation; APTT: activated partial thromboplastin time; AT: antithrombin; AUC: area under the curve; CAC: COVID-19 associated coagulopathy; COVID-19: coronavirus disease 2019; CRP: C-reactive protein; DIC: disseminated intravascular coagulation; ECMO: Extracorporeal Membranous Oxygenation; FDP: fibrin/fibrinogen degradation product ; ICU: intensive care unit; IL: interleukin; ISTH: International Society on Thrombosis and Haemostasis; JAAM: Japanese Association for Acute Medicine; JMHW: Japanese Ministry of Health and Welfare; KL: Krebs von den Lungen; NT-proBNP: N-terminal pro B-type natriuretic peptide; PAl: plasminogen activator inhibitor; PCT: procalcitonin; P-SEP: presepsin; PT-INR: PTinternational normalized ratio; ROC: receiver operating characteristic curve; RT: prothrombin time; SARSCoV : severe acute respiratory syndrome coronavirus; SF: soluble fibrin; SOFA: Sequential Organ Failure Assessment; SP-A: surfactant protein A; TAT: thrombin-antithrombin complex; TM: thrombomodulin; TNF: tumor necrosis factor; TnT: troponin T; WBC: white blood cell.

\section{Declarations}

\section{Ethics approval and consent to participate}

The following ethics review boards approved the protocol for this study: Fukuoka University Hospital (U20-10-008; registered on 22 October 2020); and Sysmex Corporation (2020-242; registered on 13 November 2020).

\section{Consent for publication}

We obtained signed informed consent from the patients or proxy in accordance with the Declaration of Helsinki for publication of this study. A copy of the informed consent document can be provided upon request.

\section{Availability of data and materials}

Should the paper be accepted, all data and materials will be available from the corresponding author (HI) and from a repository. 


\section{Competing interests}

Takahiro Yamagaito and Maho Yoshida are employees of Sysmex Corporation. The other authors declare that they have no competing interests.

\section{Funding}

This work was supported in part by a Grant of the Clinical Research Promotion Foundation 2020 (Fukuoka, Japan).

\section{Authors' contributions}

$\mathrm{HI}$ contributed to the study design, statistical analysis, interpretation of the results, drafting of the manuscript, and critical revision of the manuscript for intellectual content. JM and YN participated in designing the study and interpretating the results. MN participated in interpretating the results and drafting of the manuscript. TY and MY participated in the measurement of samples and statistical analysis. $\mathrm{KH}$ participated in designing the study and interpretating the results. TK participated in designing the study and drafting of the manuscript. SM, YI, and JN were involved in data acquisition. All authors have read and approved the final manuscript.

\section{Acknowledgments}

The authors thank Ellen Knapp, PhD, from Edanz (https://jp.edanz.com/ac) for editing a draft of this manuscript.

\section{References}

1. https://www.who.int/emergencies/disease-outbreak-news/item/2020-DON229. Accessed 8 Feb 2022.

2. https://www.who.int/director-general/speeches/detail/who-director-general-s-opening-remarks-at-themedia-briefing-on-covid-19-11-march-2020. Accessed 8 Feb 2022

3. Cascella M, Rajnik M, Aleem A, Dulebohn SC, Di Napoli R. Features, Evaluation, and Treatment of Coronavirus (COVID-19). 2021 Jul 17. In: StatPearls [Internet]. Treasure Island (FL): StatPearls Publishing; 2021 Jan-. (PMID:34033342)

4. Lopez Bernal J, Andrews N, Gower C, Gallagher E, Simmons R, Thelwall S, et al. Effectiveness of Covid-19 Vaccines against the B.1.617.2 (Delta) Variant. N Engl J Med. 2021;385:585-94. doi: 10.1056/NEJMoa2108891.

5. Bourgonje AR, Abdulle AE, Timens W, Hillebrands JL, Navis GJ, Gordijn SJ, et al. Angiotensinconverting enzyme 2 (ACE2), SARS-CoV-2 and the pathophys- iology of coronavirus disease 2019 (COVID-19). J Pathol. 2020;251:228-48. doi: 10.1002/path.5471. 
6. Li X, Ma X. Acute respiratory failure in COVID-19: is it "typical" ARDS. Crit Care. 2020;24:198. doi: 10.1186/s13054-020-02911-9.

7. Coopersmith CM, Antonelli M, Bauer SR, Deutschman CS, Evans LE, Ferrer R, et al. The Surviving Sepsis Campaign: Research Priorities for Coronavirus Disease 2019 in Critical IIIness. Crit Care Med. 2021;49:598-622. DOI: 10.1097/CCM.0000000000004895.

8. Lippi G. Sepsis biomarkers: past, present and future. Clin Chem Lab Med. 2019;57:1281-3.

9. Levi M, Thachil J, Iba T, Levy JH. Coagulation abnormalities and thrombosis in patients with COVID19. Lancet Haematol. 2020;7:e438-e440. doi:10.1016/S2352-3026(20)30145-9.

10. Connors JM, Levy JH. COVID-19 and its implications for thrombosis and anticoagulation. Blood. 2020;135:2033-40. doi:10.1182/blood.2020006000.

11. Medcalf RL, Keragala CB, Myles PS. Fibrinolysis and COVID-19: A plasmin paradox. J Thromb Haemost. 2020;18:2118-22. doi: 10.1111/jth.14960.

12. Colling ME, Kanthi Y. COVID-19-associated coagulopathy: An exploration of mechanisms. Vascular Medicine. 2020;25:471-8.

13. Lippi G, Plebani M, Henry BM. Thrombocytopenia is associated with severe coronavirus disease 2019 (COVID-19) infections: A meta-analysis. Clin Chim Acta. 2020;506:145-8. doi:

10.1016/j.cca.2020.03.022.

14. Singer M, Deutschman CS, Seymour CW, Shankar-Hari M, Annane D, Bauer M, et al. The Third International Consensus Definitions for Sepsis and Septic Shock (Sepsis-3). JAMA. 2016;315:80110. doi: 10.1001/jama.2016.0287.

15. ARDS Definition Task Force, Ranieri VM, Rubenfeld GD, Thompson BT, Ferguson ND, Caldwell E, et al. Acute respiratory distress syndrome: the Berlin Definition. JAMA. 2012;307:2526-33. doi: 10.1001/jama.2012.5669.

16. Gando S, Iba T, Eguchi Y, Ohtomo Y, Okamoto K, Koseki K, et al. A multicenter, prospective validation of disseminated intravascular coagulation diagnostic criteria for critically ill patients: comparing current criteria. Crit Care Med. 2006;34:625-31.

17. Taylor FB Jr, Toh CH, Hoots WK, Wada H, Levi M, Scientific Subcommittee on Disseminated Intravascular Coagulation (DIC) of the International Society on Thrombosis and Haemostasis (ISTH). Towards definition, clinical and laboratory criteria, and a scoring system for disseminated intravascular coagulation. Thromb Haemost. 2001;86:1327-30.

18. Kobayashi N, Maekawa T, Takada M, Tanaka H, Gonmori H. Criteria for diagnosis of DIC based on the analysis of clinical and laboratory findings in 345 DIC patients collected by the Research Committee on DIC in Japan. Bibl Haematol. 1987;49:848-52.

19. Knaus WA, Draper EA, Wagner DP, Zimmerman JE. APACHE II: a severity of disease classification system. Crit Care Med. 1985;13:818-29.

20. Vincent JL, de Mendonça A, Cantraine F, Moreno R, Takala J, Suter PM, et al. Use of the SOFA score to assess the incidence of organ dysfunction/failure in intensive care units: results of a multicenter, 
prospective study. Working group on "sepsis-related problems" of the European Society of Intensive Care Medicine. Crit Care Med. 1998;26:1793-1800.

21. Milara J, Ballester B, Montero P, Escriva J, Artigues E, Alós M, et al. MUC1 intracellular bioactivation mediates lung fibrosis. Thorax. 2020;752:132-42.

22. Ghati A, Dam P, Tasdemir D, Kati A, Sellami H, Sezgin GC, et al. Exogenous pulmonary surfactant: A review focused on adjunctive therapy for severe acute respiratory syndrome coronavirus 2 including SP-A and SP-D as added clinical marker. Curr Opin Colloid Interface Sci. 2021;51:101413. doi:

10.1016/j.cocis.2020.101413.

23. Gao L, Jiang D, Wen XS, Cheng XC, Sun M, He B, et al. Prognostic value of NT-proBNP in patients with severe COVID-19. Respir Res. 2020;21:83. doi: 10.1186/s12931-020-01352-w.

24. Guo T, Fan Y, Chen M, Wu X, Zhang L, He T, et al. Cardiovascular Implications of Fatal Outcomes of Patients With Coronavirus Disease 2019 (COVID-19). JAMA Cardiol. 2020;5:811-8. doi: 10.1001/jamacardio.2020.1017.

25. Hanley JA, McNeil BJ. The meaning and use of the area under a receiver operating characteristic (ROC) curve. Radiology. 1982;143:29-36.

26. Terpos E, Ntanasis-Stathopoulos I, Elalamy I, Kastritis E, Sergentanis TN, Politou M, et al. Hematological findings and complications of COVID-19. Am J Hematol. 2020;95:834-47. doi:10.1002/ ajh.25829.

27. Zaninotto M, Mion MM, Cosma C, Rinaldi D, Plebani M. Presepsin in risk stratification of SARS-CoV-2 patients. Clin Chim Acta. 2020;507:161-3. doi: 10.1016/j.cca.2020.04.020.

28. Maesaki S, Maeda T. Presepsin as a predictive biomarker of severity in COVID-19: a case series. J Med Virol. 2021;93:99-101. doi: 10.1002/jmv.26164.

29. Schirinzi A, Cazzolla AP, Lovero R, Lo Muzio L, Testa NF, Ciavarella D, et al. New Insights in Laboratory Testing for COVID-19 Patients: Looking for the Role and Predictive Value of Human epididymis secretory protein 4 (HE4) and the Innate Immunity of the Oral Cavity and Respiratory Tract. Microorganisms. 2020;8:1718. doi: 10.3390/microorganisms8111718.

30. Chen G, Wu D, Guo W, Cao Y, Huang D, Wang H, et al. Clinical and immunological features of severe and moderate coronavirus disease 2019. J Clin Invest. 2020;130:2620-9. doi: 10.1172/JCI137244.

31. Scully M, Levi M. How we manage haemostasis during sepsis. Br J Haematol. 2019;185:209-18. doi: 10.1111/bjh.15821.

32. Akca S, Haji-Michael P, de Mendonça A, Suter P, Levi M, Vincent JL. Time course of platelet counts in critically ill patients. Critical Care Medicine 2002;30:753-6.

33. Van derschueren S, DeWeerdt A, Malbrain M, Vankersschaever D, Frans E, Wilmer A, et al. Thrombocytopenia and prognosis in intensive care. Critical Care Medicine. 2000;28:1871-6.

34. Zhang Y, Zeng X, Jiao Y, Li Z, Liu Q, Ye J, et al. Mechanisms involved in the development of thrombocytopenia in patients with COVID-19. Thromb Res. 2020;193:110-5. doi:

10.1016/j.thromres.2020.06.008. 
35. Al-Samkari H, Karp Leaf RS, Dzik WH, Carlson JCT, Fogerty AE, Waheed A, et al. COVID-19 and coagulation: bleeding and thrombotic manifestations of SARS-CoV-2 infection. Blood. 2020;136:489-500. doi: 10.1182/blood.2020006520.

36. Furusako S, Shirakawa K. Methods for detecting human low molecular weight CD14. United States patent 2008; US7465547 B2.

37. Furusako S, Shirakawa K, Hirose J. Soluble CD14 antigen. United States patent 2009; US7608684 B2.

38. Lippi G, Cervellin G. Can presepsin be used for screening invasive fungal infections? Ann Transl Med. 2019;7:87. doi: 10.21037/atm.2019.01.40.

39. Shozushima T, Takahashi G, Matsumoto N, Kojika M, Okamura Y, Endo S. Usefulness of presepsin (sCD14-ST) measurements as a marker for the diagnosis and severity of sepsis that satisfied diagnostic criteria of systemic inflammatory response syndrome. J Infect Chemother. 201117:7649.

40. Endo S, Suzuki Y, Takahashi G, Shozushima T, Ishikura H, Murai A, et al. Usefulness of presepsin in the diagnosis of sepsis in a multicenter prospective study. J Infect Chemother. 2012;18:891-7. doi: 10.1007/s10156-012-0435-2.

41. Ishikura H, Nishida T, Murai A, Nakamura Y, Irie $Y$, Tanaka J, et al. New diagnostic strategy for sepsisinduced disseminated intravascular coagulation: a prospective single-center observational study. Crit Care. 201418: R19. doi: 10.1186/cc13700.

42. Lu B, Zhang Y, Li C, Liu C, Yao Y, Su M, et al. The utility of presepsin in diagnosis and risk stratification for the emergency patients with sepsis. Am J Emerg Med. 2018;36:1341-5. doi: 10.1016/j.ajem.2017.12.038.

43. Dell'Aquila P, Raimondo P, Orso D, De Luca P, Pozzessere P, Parisi CV, et al. A simple prognostic score based on troponin and presepsin for COVID-19 patients admitted to the emergency department: a single-center pilot study. Acta Biomed. 2021;92:e2021233. doi: 10.23750/abm.v92i4.11479.

44. Lippi G, Sanchis-Gomar F, Henry BM. Presepsin value predicts the risk of developing severe/critical COVID-19 illness: results of a pooled analysis. Chem Lab Med. 2021 Sep 2. doi: 10.1515/cclm-20210848. Online ahead of print.

45. Yamaguchi H, Takuma A, Fukuoka E, Oto H, Inoue M, Nagahama T, et al. Concentration of Soluble CD14-Subtype (Presepsin) in Plasma of Non-Inflammatory and Septic Febrile Children Rinsho Byori. 2016;64:1001-6. [Article in Japanese]

46. Tavazzi G, Pellegrini C, Maurelli M, Belliato M, Sciutti F, Bottazzi A, et al. Myocardial localization of coronavirus in COVID-19 cardiogenic shock. Eur J Heart Fail. 2020;22:911-5. doi: 10.1002/ejhf.1828.

47. Linscheid P, Seboek D, Nylen ES, Langer I, Schlatter M, Becker KL, et al. In vitro and in vivo calcitonin I gene expression in parenchymal cells: a novel product of human adipose tissue. Endocrinology. 2003;144:5578-84. doi: 10.1210/en.2003-0854.

48. Schwarz S, Bertram M, Schwab S, Andrassy K, Hacke W. Serum procalcitonin levels in bacterial and abacterial meningitis. Crit Care Med. 2000;28:1828-32. doi: 10.1097/00003246-200006000-00024. 
49. Han H, Ma Q, Li C, Liu R, Zhao L, Wang W, et al. Profiling serum cytokines in COVID-19 patients reveals IL-6 and IL-10 are disease severity predictors. Emerg Microbes Infect. 2020;9:1123-30. doi: 10.1080/22221751.2020.1770129.

50. Alkholi UM, Abd Al-Monem N, Abd El-Azim AA, Sultan MH. Serum procalcitonin in viral and bacterial meningitis. J Glob Infect Dis. 2011;3:14-8. doi: 10.4103/0974-777X.77290.

51. Henry BM, Benoit SW, de Oliveira MHS, Hsieh WC, Benoit J, Ballout RA, et al. Laboratory abnormalities in children with novel coronavirus disease 2019. Clin Biochem. 2020;81:1-8. doi: 10.1016/j.clinbiochem.2020.05.012.

52. Lippi G, Plebani M. Procalcitonin in patients with severe coronavirus disease 2019 (COVID-19): A meta-analysis. Clin Chim Acta. 2020;505:190-1. doi: 10.1016/j.cca.2020.03.004

53. Hu R, Han C, Pei S, Yin M, Chen X. Procalcitonin levels in COVID-19 patients. Int J Antimicrob Agents. 2020;56:106051. doi: 10.1016/j.jjantimicag.2020.106051.

54. Tajbakhsh A, Gheibi Hayat SM, Taghizadeh H, Akbari A, Inabadi M, Savardashtaki A, et al. COVID-19 and cardiac injury: clinical manifestations, biomarkers, mechanisms, diagnosis, treatment, and follow up. Expert Rev Anti Infect Ther. 2021;19:345-57. doi: 10.1080/14787210.2020.1822737.

55. Zhao Y, Qin L, Zhang P, Li K, Liang L, Sun J, et al. Longitudinal COVID-19 profiling associates IL-1RA and IL-10 with disease severity and RANTES with mild disease. JCI Insight. 2020;5:e139834. doi: 10.1172/jci.insight.139834.

56. Li X, Xu S, Yu M, Wang K, Tao Y, Zhou Y, et al. Risk factors for severity and mortality in adult COVID19 inpatients in Wuhan. J Allergy Clin Immunol. 2020;146:110-8. doi: 10.1016/j.jaci.2020.04.006.

57. Huang C, Wang Y, Li X, Ren L, Zhao J, Hu Y, et al. Clinical features of patients infected with 2019 novel coronavirus in Wuhan, China. Lancet. 2020;395:497-506. doi: 10.1016/S0140-6736(20)301835 .

58. Han H, Ma Q, Li C, Liu R, Zhao L, Wang W, et al. Profiling serum cytokines in COVID- 19 patients reveals IL-6 and IL-10 are disease severity predictors. Emerg Microbes Infect. 2020;9:1123-30. doi: 10.1080/22221751.2020.1770129.

59. Diao B, Wang C, Tan Y, Chen X, Liu Y, Ning L, et al. Reduction and functional exhaustion of T cells in patients with coronavirus disease 2019 (COVID-19). Front Immunol. 2020;11:827. doi: 10.3389/fimmu.2020.00827. eCollection 2020.

60. Wang F, Hou H, Luo Y, Tang G, Wu S, Huang M, et al. The laboratory tests and host immunity of COVID-19 patients with different severity of illness. JCI Insight. 2020;5:e137799. doi: 10.1172/jci.insight.137799.

61. Zhao Y, Qin L, Zhang P, Li K, Liang L, Sun J, et al. Longitudinal COVID-19 profiling associates IL-1RA and IL-10 with disease severity and RANTES with mild disease. JCI Insight. 2020;5:e139834. doi: 10.1172/jci.insight.139834.

62. Lu L, Zhang H, Dauphars DJ, He YW. A Potential Role of Interleukin 10 in COVID-19 Pathogenesis. Trends Immunol. 2021;42:3-5. doi: 10.1016/j.it.2020.10.012. Epub 2020 Nov 2. 
63. Ali MAM, Spinler SA. COVID-19 and thrombosis: From bench to bed side. Trends Cardiovasc Med. 2021;31:143-60. doi: 10.1016/j.tcm.2020.12.004.

64. Chan NC, Weitz JI. COVID-19 coagulopathy, thrombosis, and bleeding. Blood. 2020;136:381-3. doi: 10.1182/blood.2020007335.

\section{Tables}

Tables 1-3 are available in the Supplemental Files section.

\section{Figures}

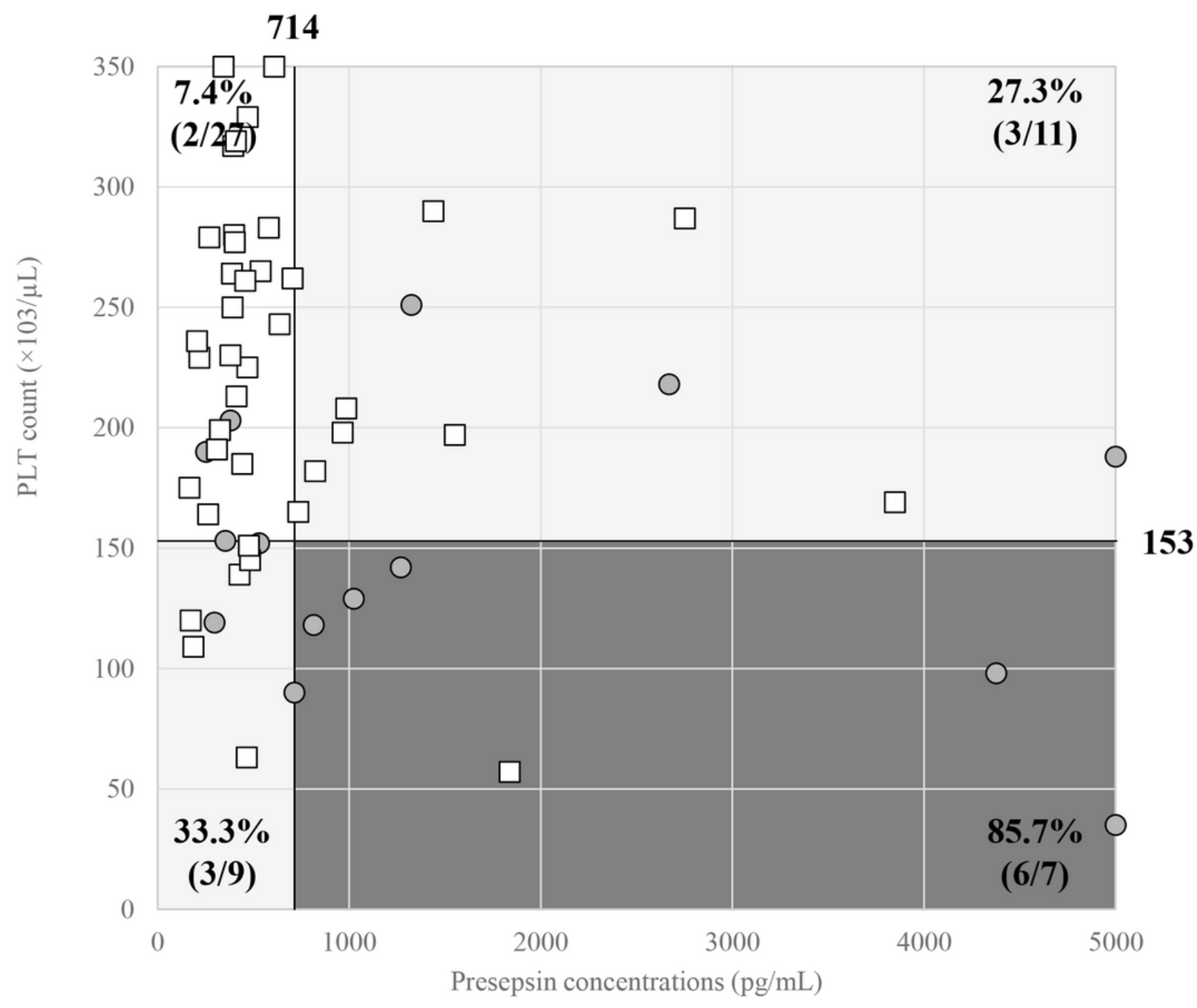

Figure 1

Severity and classification panel of patients with serious COVID-19 patients. Patients were classified into the following four panels according to the cay-off value from platelet count and Presepsin levels: (1) 
platelet count $\leq 153 \times 10^{3} / \mu \mathrm{L}$ and P-SEP $\geq 714 \mathrm{pg} / \mathrm{mL}$; (2) platelet count $>153 \times 10^{3} / \mu \mathrm{L}$ and P-SEP $\geq 714$ $\mathrm{pg} / \mathrm{mL}$, (3) platelet count $\leq 153 \times 10^{3} / \mu \mathrm{L}$ and P-SEP $<714 \mathrm{pg} / \mathrm{mL}$ and (4) platelet count $>153 \times 10^{3} / \mu \mathrm{L}$ and P-SEP $<714 \mathrm{pg} / \mathrm{mL}$. And classification of serious COVID-19 patients. The categories were classified as follows: (A) High risk group, dark gray area (1); (B) Medium risk group, gray area (2) + (3); and (C) Low risk group, white area (4). And the in-hospital mortality of the high-risk, medium-risk, and low-risk groups was $85.7 \%(6 / 7), 30.0 \%(6 / 20)$, and $7.4 \%(2 / 27)$, respectively.

COVID, coronavirus disease; PLT, platelet.

APACHE II score

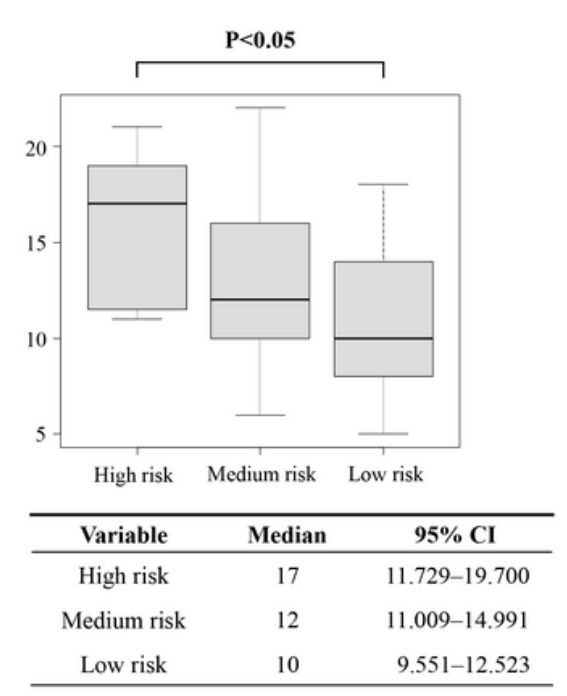

SOFA score

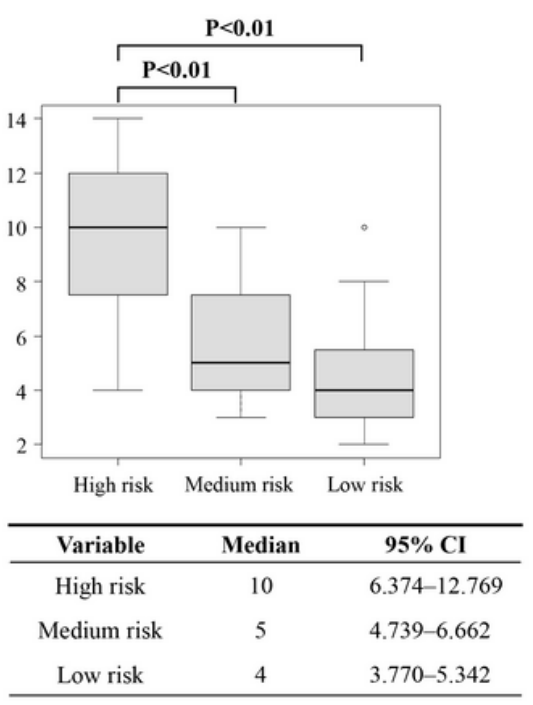

Age

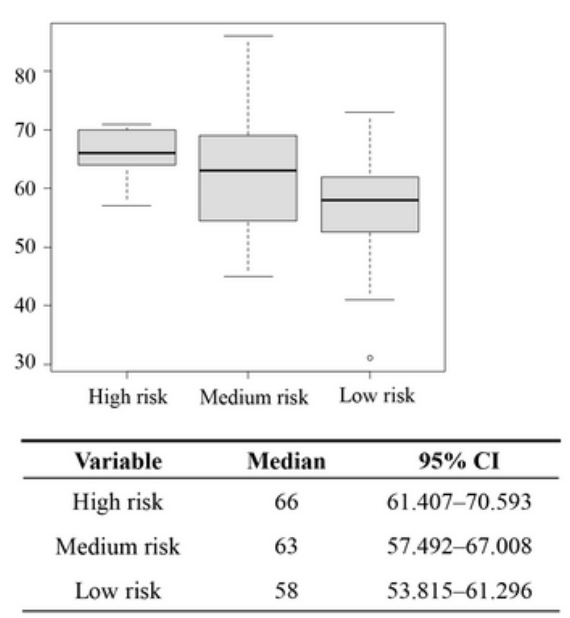

\section{Figure 2}

Comparison of APACHE II score, SOFA score, and age for each severity group classified by platelet count and presepsin. When patients were categorized 3 groups according to the severity defined in the panel (see Figure 1), the APACHE II score, and the SOFA score on ICU admission significantly increased as the COVID-19 severity increased. But age was no significant difference between three groups. However, the age increased as the risk of the group increased. The Bonferroni Method was used to compare between three groups, High risk, Medium risk, and Low risk. 


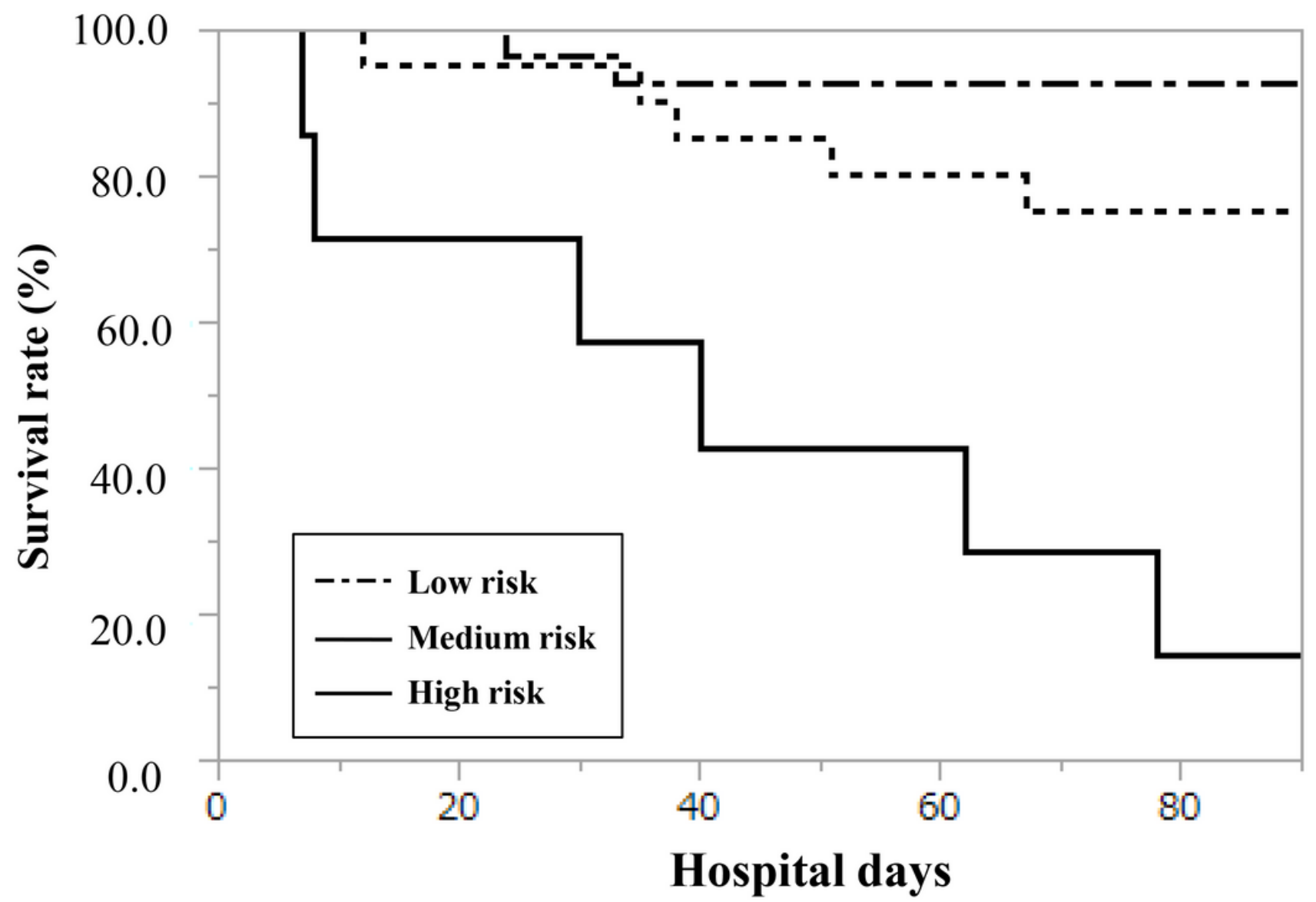

Figure 3

Kaplan--Meier curve for 90-day survival stratified by severity. Kaplan-Meier survival plots for survival stratified by severity for 90-day period. The survival rate was significantly higher in the Low risk group than in the both Moderate risk and High risk group. ( $P<0.01$ by Log-rank test). 

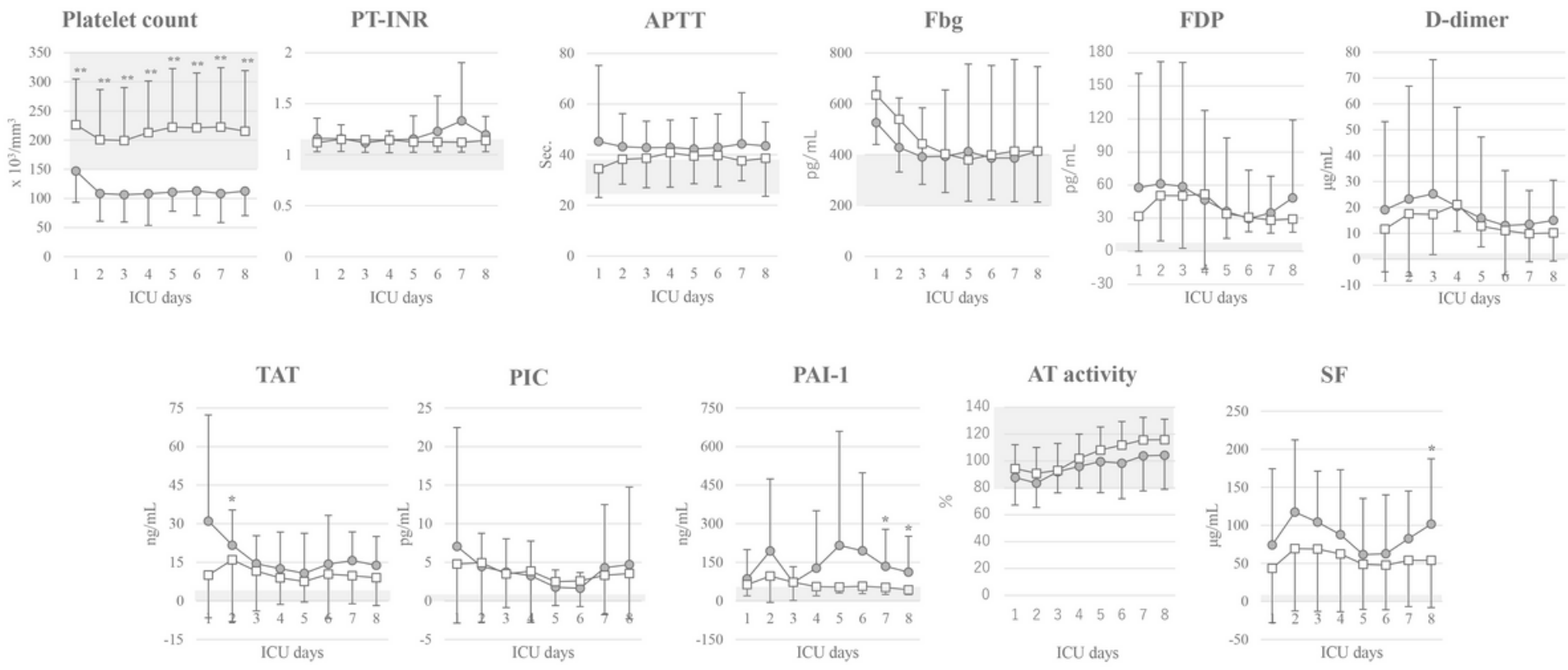

\section{Figure 4}

Daily changes in coagulation/fibrinolysis markers for each outcome. Open square indicates Survivor group, and closed circle indicates Non-survivor group. Only platelet counts were significantly lower in the non-survivor group than in the survivor group on all days throughout the 8 days. ${ }^{*} P<0.05,{ }^{\star \star} P<0.01$, between the linked groups. The gray area indicates the normal range.
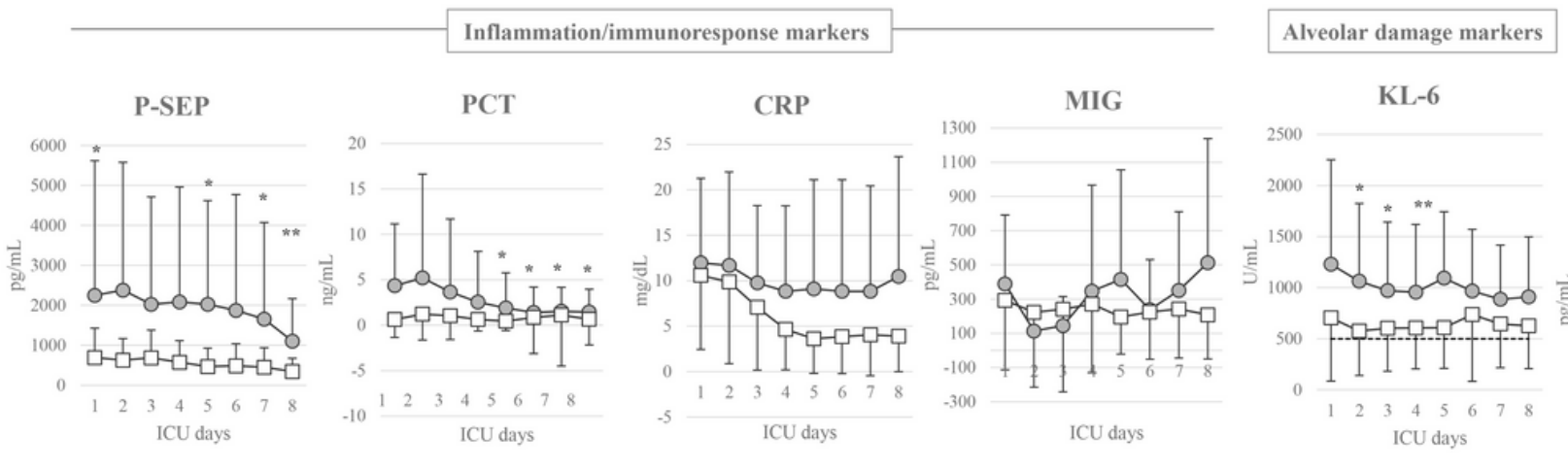

Myocardial damage markers

IL-6

IL-10

IL-18
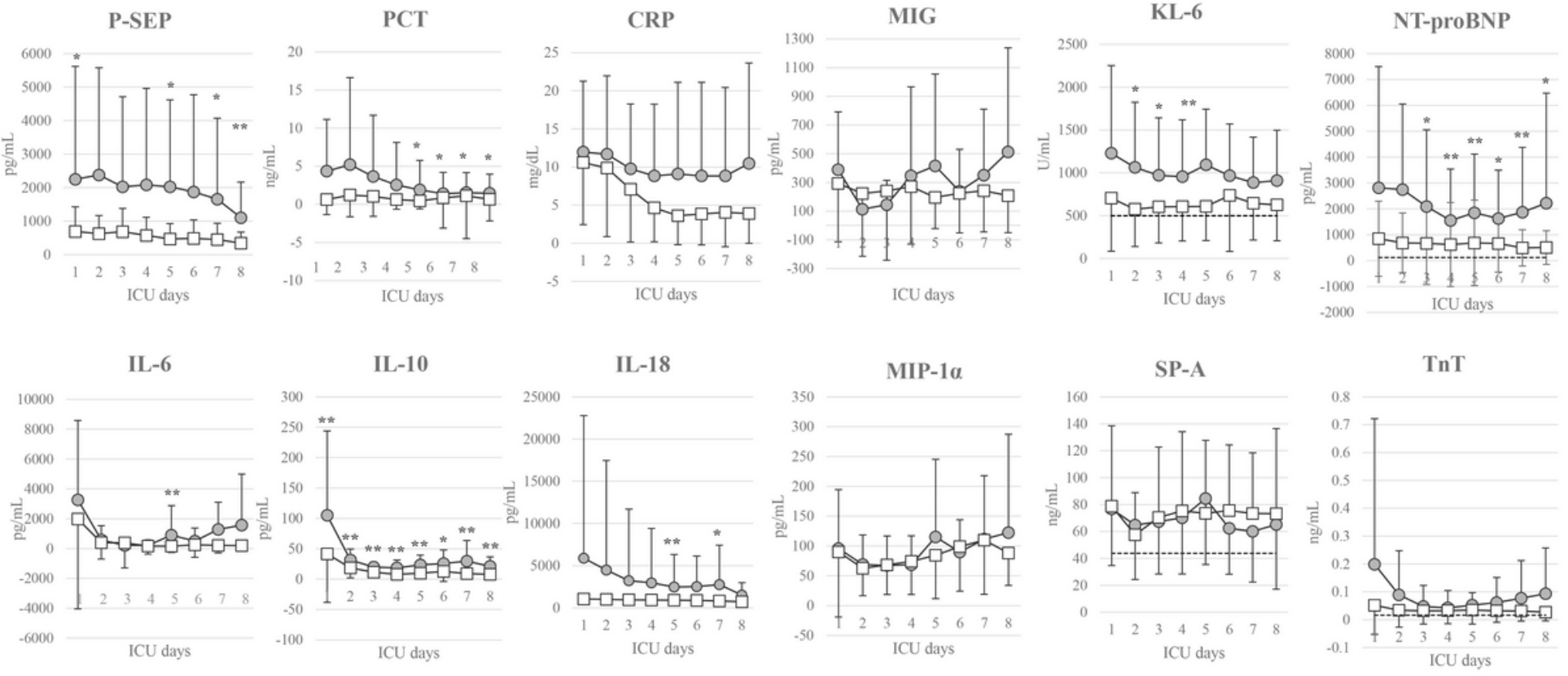


\section{Figure 5}

Daily changes in inflammation/immunoresponse markers and alveolar/myocardial damage markers for each outcome. Open square indicates Survivor group, and closed circle indicates Non-survivor group. In inflammation/immunoresponse markers only IL-10 was significantly higher in the non-survivor group than in the survivor group on all days throughout the 8 days. P-SEP was significantly higher in the nonsurvivor group than in the survivor group in 4 of the 8 days, excluding Day 2, 3, 4 and 6 . PCT was also significantly higher in the non-survivor group than in the survivor group in 4 of the 8 days, excluding initial 4 days of Day 1 to Day 4. In alveolar/myocardial damage markers, NT-proBNPproBNP concentrations were significantly higher in the non-survivor group than in the survivor group in 6 of the 8 days, excluding Day 1 and 2 . ${ }^{*} P<0.05,{ }^{*} P<0.01$, between the linked groups.

\section{Supplementary Files}

This is a list of supplementary files associated with this preprint. Click to download.

- SupplementaryfileSupplementTable12.pdf

- Table13.pdf 